\title{
Radiation and immunotherapy: emerging mechanisms of synergy
}

\author{
William G. Breen ${ }^{1}$, Konstantinos Leventakos ${ }^{2}$, Haidong Dong ${ }^{3}$, Kenneth W. Merrell ${ }^{1}$ \\ ${ }^{1}$ Department of Radiation Oncology, Mayo Clinic, Rochester, MN, USA; ${ }^{2}$ Division of Medical Oncology, Mayo Clinic, Rochester, MN, USA; \\ ${ }^{3}$ Departments of Urology and Immunology, Mayo Clinic College of Medicine and Science, Rochester, MN, USA \\ Contributions: (I) Conception and design: WG Breen, KW Merrell; (II) Administrative support: None; (III) Provision of study materials or patients: \\ None; (IV) Collection and assembly of data: None; (V) Data analysis and interpretation: None; (VI) Manuscript writing: All authors; (VII) Final \\ approval of manuscript: All authors. \\ Correspondence to: Kenneth W. Merrell, MD. Department of Radiation Oncology, Mayo Clinic, 200 First Street SW, Rochester, MN 55905, USA. \\ Email: Merrell.Kenneth@mayo.edu.
}

\begin{abstract}
Immunotherapy (IO) has become a standard treatment in patients with metastatic and locally advanced non-small cell lung cancer (NSCLC), and is now being tested in patients with early stage disease. IO agents currently in use for lung cancer target PD-1, PD-L1, and CTLA-4. While survival and tumor control have improved with IO, many patients have limited or short responses to IO. Therefore, methods to improve the systemic response to IO are needed. Radiation therapy (RT) is an integral component of lung cancer treatment, and may improve systemic response to IO by increasing antigen presentation, increasing co-stimulatory signaling, increasing T-cells recruitment, upregulating PD-L1, increasing tumor stromal lymphocyte infiltration, and altering the microenvironment. IO after definitive chemoradiation is now standard treatment in unresectable stage III NSCLC following publication of the PACIFIC clinical trial. For early stage NSCLC, IO is being investigated in conjunction with stereotactic body radiotherapy (SBRT). The benefit of adding RT to IO in patients with metastatic disease may be especially pronounced in patients with low baseline PD-L1 expression, potentially when delivered as a short course of SBRT, as supported by the PEMBRO-RT clinical trial. Current and ongoing clinical trials are evaluating the optimal radiation dose, timing, and sequencing of RT with IO.
\end{abstract}

Keywords: Radiation therapy (RT); immunotherapy (IO); SBRT; lung cancer; metastatic disease

Submitted Apr 08, 2020. Accepted for publication May 04, 2020.

doi: $10.21037 /$ jtd-2019-cptn-07

View this article at: http://dx.doi.org/10.21037/jtd-2019-cptn-07

\section{Introduction}

Knowledge of the immune system's interactions with cancer cells and the ability of therapeutics to harness these interactions have substantially improved in recent years. For patients with lung cancer, treatment paradigms, tumor control, and survival are quickly evolving in the era of immunotherapy (IO). Still, even among cancers that initially respond to IO, most eventually develop resistance and progress. Radiation therapy (RT) plays an important role in the treatment of lung cancer, and may help to overcome tumor resistance to IO through a variety of mechanisms. In this review, we aim to describe the evidence for the evolving role and synergistic mechanisms of combining IO with RT, including the optimal dose, volume, and timing of radiation for effective immune stimulation.

\section{Cancer immunology}

\section{The immune synapse}

Immune responses are regulated by the elaborate interactions between T-cells and antigen presenting cells (APC), referred to as the immune synapse. With appropriate regulation within the host, immune responses provide defense against pathogens and aberrant cellular growth. Cytoactivity of T-cells is regulated by both the presence and spatial alignment of co-stimulatory or -inhibitory signals 
and receptors, and is further regulated by expression of cytokines. While a complete summary of these processes is beyond the scope of this review, co-stimulatory signals lead to activation of an immune response, whereas the absence of co-stimulatory or the presence of co-inhibitory signals result in the lack of an immune response, or anergy.

The following are immune cells involved in the process of tumor recognition and response (1):

(I) T lymphocytes: $\mathrm{CD} 8^{+}$and $\mathrm{CD}^{+}$lymphocytes, commonly referred to as cytotoxic T-cells and helper T-cells, are involved in the distinction of self and non-self antigens through interactions with T-cell receptors (TCR) and major histocompatibility complex (MHC) proteins.

(II) T regulatory cells (TReg): TReg's inhibit T-cell activation. Other immune suppressive cells include myeloid derived suppressor cells (MDSCs).

(III) Natural Killer (NK) cells: NK cells target cells with low MHC class 1 expression for elimination. Unlike T-cells, NK cells do not require antigen presentation. Similar to T-cells, they may secrete numerous inhibitor signals.

(IV) Macrophages: Phagocytes involved in cell clearance and secretion of interferons and other cytokines. M1 macrophages perform phagocytosis and release interferons. M2 macrophages release cytokines and TGF-beta and are inhibitor regulators involved with immune tolerance.

(V) Antigen presenting cells (APC): APC's mediate immune response through processing and presenting of antigens to T-Cells, and release of cytokines.

Control and regulation of the immune response is paramount to limiting the impact on normal host tissue. Dysregulation of immune responses may lead to autoimmune effects and other medical disorders. Two critical pathways of co-inhibitor immune checkpoint are clinically relevant to cancer IO:

\section{Cytotoxic T lymphocyte antigen 4 (CTLA-4)}

CTLA-4 is a fundamental inhibitory regulator of T-cell immune response. CTLA-4 has inducible expression with naïve T-cell activation, and inhibits immune activation (2). CTLA-4 is homolog of CD28 (T-cell co-stimulatory receptor) present on T-cells and binds to the same ligands as CD28 (CD80 and CD86) on APC but with a higher affinity. Therefore, a major pathway of T-cell inhibition of CTLA4 is through outcompeting CD28 for binding with CD80 and CD86 $(3,4)$. Expression of CTLA-4 is driven by TCR activation as well as IL-12 and IFN gamma. Heterozygous CTLA-4 mutations result in dysregulation of T-cells, with subsequent hyperproliferation and organ infiltration, and have been implicated in auto-immune disorders. Supporting these findings, CTLA-4 knockout mice show multi-organ auto-immunity with severe disease (5).

\section{Program cell death protein 1 (PD-1)}

$\mathrm{PD}-1$ is a major regulator of the immune system and controls many T-cell functions including activation, tolerance, and exhaustion (2). PD-1 is a transmembrane receptor found in multiple cells including T-cells, NK cells and $\mathrm{B}$ cells. PD-1 has two known ligands: PD-L1 and PDL2. PD-L1 is more widely expressed in both hematopoietic and non-hematopoietic cells, including tumor cells, and is induced via pro-inflammatory cytokines $(6,7)$. Engagement of PD-1 by PD-L1 and PD-L2 may lead to cessation of the immune response and serve as a negative feedback to limit subsequent organ damage. Therefore, when PD-1 or PDL1 is inhibited, T-cells can be activated. Similar to CTLA-4 knockout, PD-1 knockout leads to autoimmunity, but with a milder phenotype (8).

\section{Cancer immune evasion and immunotherapy}

Cancer cells are immunologically distinct from host cells, in that they may express unique non-self oncoviral or neopeptide antigens, rendering themselves detectable to T-cells, and prone to subsequent elimination. Immunosurveillance is the process by which the immune system surveys, detects, and destroys aberrant cells, including pathogens and malignant cells. The regulatory process of immune inhibition is involved in cancer progression and growth through a process described as immunoediting, in which the immune system can both constrain and promote cancer progression.

Three phases are present in immunoediting: elimination, equilibrium, and escape (9). Elimination is the process of the innate and adaptive immune system detecting and eliminating malignant processes before they become clinically apparent. When successful, elimination leads to complete eradication of cancer: cure. Equilibrium and escape may function sequentially or independently. Equilibrium occurs when cancer cells survive the elimination phase, but are prevented from progression, remaining in a dormant state with outgrowth specifically controlled by immunity. Eventually, patients may experience 
a late local or distant recurrence due to (I) antigen loss (II) insensitivity to effector mechanisms and/or (III) induction of an immunosuppressive tumor microenvironment. This leads to the escape phase, in which tumor progression and growth are no longer inhibited.

A high quantity of genetic mutations within a tumor, referred to as the tumor mutational burden (TMB), is a characteristic feature of many human cancers as quantified through whole-genome and exome sequencing $(10,11)$. Aberrant cellular pathways of DNA replication and damage repair lead to an unstable genome and high rate of somatic mutations from replicative and environmental damage. Tumors with a large number of mutations are more likely to express novel neo-peptides with subsequent immune activation. Over time, and through direct evolutionary pressure from tumor killing by the immune system, cancer cells with the ability to evade the immune system are selected for. This allows for tumor growth and progression unchecked by the immune system. Tumor cells promote an immunosuppressive microenvironment via cytokine secretion or recruitment of regulatory immune cells, such as TReg and myeloid suppressor cells, which in turn inhibit tumor specific T-cells by expressing negative co-stimulatory CTLA-4, PD-1 and PD-L1, among other mechanisms.

PD-L1 expressed on tumor cells may inhibit T-cell activation through direct binding of PD-1 on T-cells, inhibiting tumor cell killing (12). In the pre-IO era, high PD-L1 tumor proportion score (TPS) was a poor prognostic factor, associated with worse overall survival (OS) (13). PD-1 and PD-L1 inhibitors work to counteract this immune escape mechanism of cancer cells, and have revolutionized the treatment of lung cancer. The mechanisms of PD-1, PD-L1, and CCTLA-4 inhibitors are demonstrated in Figure 1.

To date, there are $4 \mathrm{IO}$ agents with FDA approval (Table 1) for clinical use in lung cancer, and numerous clinical trials evaluating the efficacy of IO. While IO has provided unprecedented survival and cancer control for many lung cancer patients, the majority will still eventually experience progressive disease. Mechanisms to overcome tumor equilibrium and escape are needed, and in some cases may be provided by RT.

\section{Immune effects of RT for non-small cell lung cancer (NSCLC)}

RT plays a key role in curative and palliative intent treatment of lung cancer. The primary anti-neoplastic effect of RT is double stranded DNA break, which ultimately leads to cell death. Cell death may occur through apoptosis, necrosis, autophagy, mitotic catastrophe, and proliferative senescence. During this process of cellular damage and death, several radiation-induced immune processes occur which have the potential to mediate an anti-neoplastic immune response, via the following mechanisms (Figure 2) (14-24):

(I) Costimulatory immune signaling with cytokines and chemokines activating and recruiting T-cells to tumor sites;

(II) Increased cancer antigen spillage, epitope diversity, and antigen presentation;

(III) Increased vascular adhesion molecule-1 promoting extravasation of T-cells;

(IV) Increased lymphocyte infiltration into the tumor stroma;

(V) Alteration of the tumor microenvironment;

(VI) Upregulation of PD-L1 on cancer cells;

(VII) Utilization of the Fas/FasL and other pathways, promoting elimination of tumor cells.

Through these processes, radiation may function as an in situ vaccination specific to the patient's genetics and biology, ultimately leading to enhanced immune recognition and cancer killing. In some cases, treatment of a localized tumor may result in distant tumor regression. This process is coined the "abscopal effect" ('ab' - away from, 'scopus' - target) (25). In theory, localized radiation treatment to a single site of disease, such as palliative RT to a painful metastasis, could lead to a systemic immunologic response with improved cancer cell recognition and subsequent regression of non-irradiated metastatic disease or occult metastatic disease in non-metastatic patients (Figure 2). The frequency of the abscopal effect in metastatic patients from $\mathrm{RT}$ alone is unclear, but is presumed to be relatively rare, with most of the literature limited to small case series and anecdotes $(25,26)$. A single clinical trial evaluated RT with granulocyte-macrophage colony-stimulating factor as a proof of principal study and detected a $26.8 \%$ incidence of distant response in patients with metastatic, solid tumors (27).

The addition of Durvalumab after definitive chemoradiation (CRT) for stage III lung cancer resulted in a reduction in the incidence of metastatic lesions by $33 \%$, ultimately leading to improvement in OS (28). The combination of RT with IO may lead to an increased likelihood of abscopal responses, and also synergistically lead to improved IO response by the mechanisms listed above. The continued elucidation of the biological interactions between RT and IO will be crucial in understanding how to 

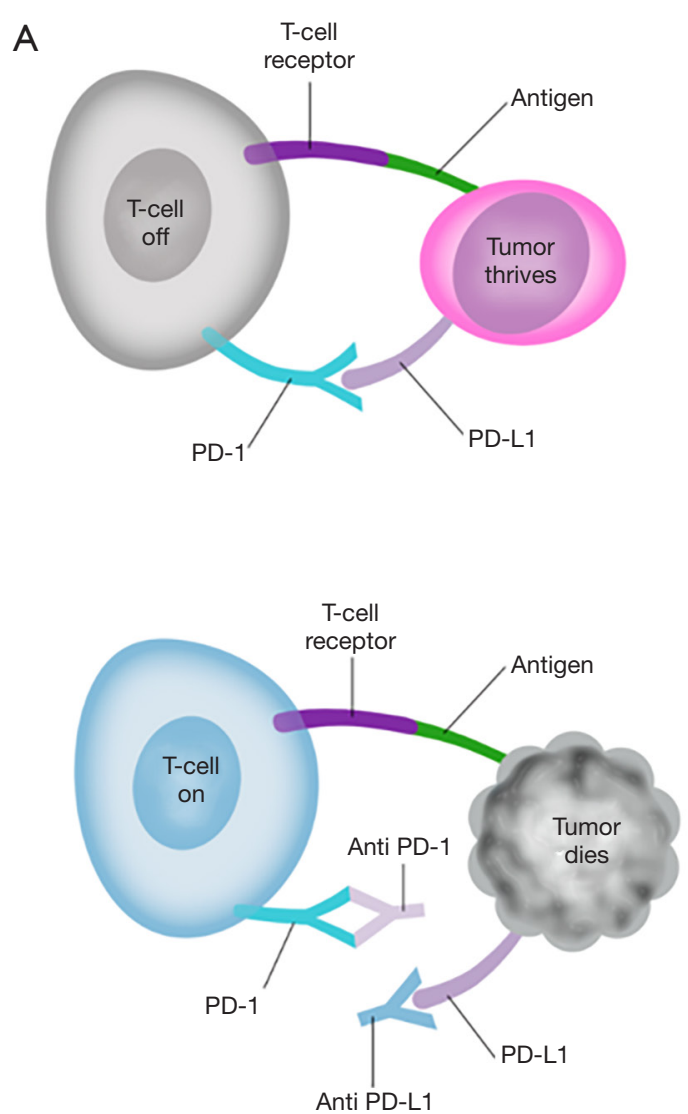
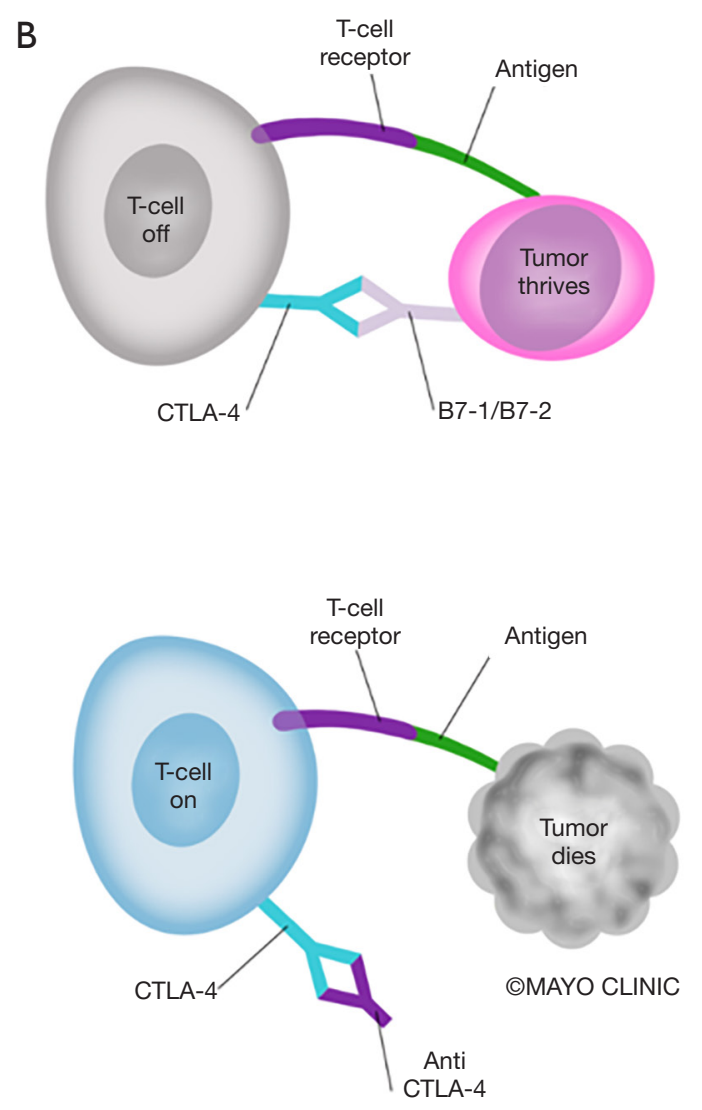

Figure 1 Mechanisms of Action of Immunotherapy Agents. Anti PD-1 and PD-L1 (A) and Anti CTLA-4 (B) antibodies facilitate T-cell mediated kill of tumor cells.

use RT to optimize IO efficacy.

\section{Optimizing radiation therapy}

\section{Radiation dose, fractionation and volume}

Modern RT planning and delivery for lung cancer is increasingly sophisticated and individualized for each patient. The dose, fractionation, and volume of irradiation depend upon several factors including tumor histology, stage, anatomy, and treatment intent. Patients undergoing curative intent treatment for locally advanced NSCLC often receive conventionally fractionated RT of 2 Gy per day over 6 weeks, along with concurrent cytotoxic chemotherapy. Patients undergoing palliative intent RT commonly receive regimens ranging from $8 \mathrm{~Gy}$ in 1 fraction to $30 \mathrm{~Gy}$ in 10 fractions. For patients with early stage inoperable NSCLC, RT may be delivered in as few as 3 high-dose fractions of 18 Gy of stereotactic radiation body therapy (SBRT). In these patients, SBRT provides superior outcomes compared to conventionally fractionated RT (29).

An important challenge in harnessing the immunostimulatory potential of RT is determining the optimal dose and fractionation. Immune effector cells are exquisitely sensitive to RT, with an LD50 (lethal dose required to half the surviving fraction of lymphocytes) of only 2 Gy (30). This estimate would translate to $50 \%$ of lymphocytes within the target volume being killed after each of the 30 fractions of a conventional course of definitive RT. Repetitive killing of immune cells with fractionated RT may erase the potential for RT-mediated immune activity over time, as several studies correlate treatment induced lymphopenia with worse overall prognosis (31-33). Therefore, despite the larger dose of RT per fraction, hypofractionated RT or SBRT may have less detriment on immune cell killing over the total course of RT compared to conventionally fractionated RT, with the potential for improved synergy between RT and IO (34).

Another consideration related to RT dose is the impact on the tumor micro-environment and immunostimulation. 
Table 1 PD-1/PD-L1 inhibitors with labeled indication for non-small cell lung cancer (NSCLC)

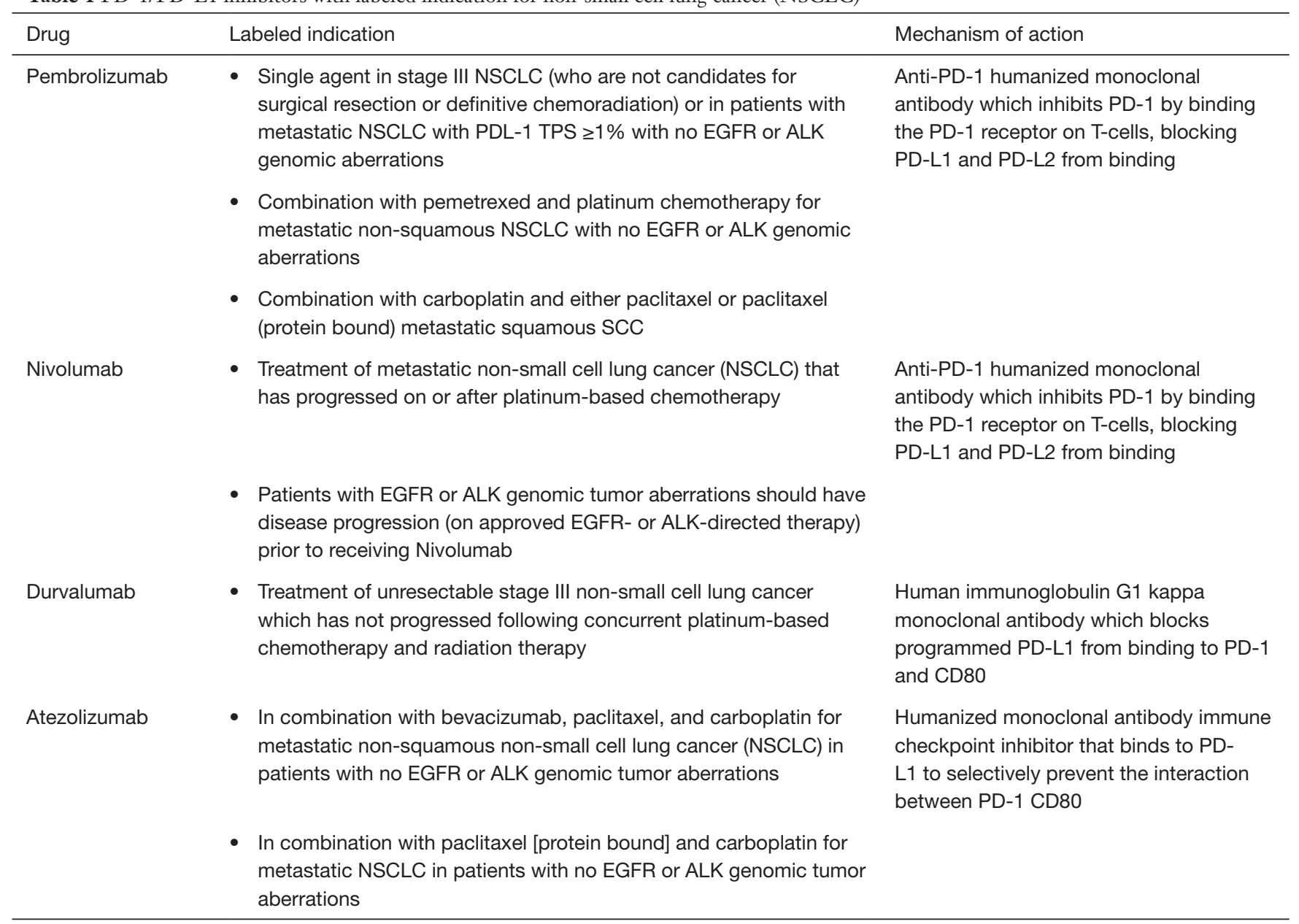

Extensive tumor infiltration by T-cells is correlated with improved survival in NSCLC patients (35). SBRT results in a greater degree of stromal and vascular damage and tumor apoptosis compared to conventional fractionation (36). SBRT increases the potential for greater antigen spillage, presentation, and recognition through increased generation and trafficking of anti-tumor effector cells to the tumor site (37). This process may be synergistic to both CTLA4 and PDL-1 pathway inhibition (38). In animal models, 5 fractions of 6 Gy and 3 fractions of 8 Gy have shown immunemediated abscopal effects in mice treated with IO (39). Interestingly, this study also indicated better responses for patients receiving multiple fractions of RT compared to a single fraction, potentially due to increased number and diversity of tumor neo-antigens produced after multiple fractions of radiation. Similar dose and fractionation schemes have been used in human studies, with results indicating safety, and efficacy data under evaluation (40-42).

While most trials of IO and RT are evaluating high doses of RT per fraction for immunostimulation, some are also evaluating whether an ultra-low dose of RT per fraction may improve the immunogenic response to RT. On one analysis, a dose of $0.5 \mathrm{~Gy}$ was more effective in recruiting T-cell infiltration in tumors as measured 7 days after RT than 1, 2, or 6 Gy fractions (43). Additional previous studies have shown low-dose RT to be a potentiator of cisplatin in lung cancer cells (44). An ongoing clinical trial is comparing high dose RT $(8 \mathrm{~Gy} \times 3)$ to low-dose fractionated RT (0.5 Gy BID $\times 2$ days, $\mathrm{q} 4$ weeks $\times 4$ ), with both arms receiving concurrent IO (NCT02888743).

As oncologists work to find the optimal immunostimulatory dose of radiation, they must also consider the ideal volume and/or number of lesions to treat. While most clinical trials investigating IO and RT completed to date dictated 

A Immunogenic Impactof RT
- Increased cancer antigen presentation
- T cell activation/recruitment
- Alteration of the tumor microenvironment
- Upregulation of PD-L1
- Increased recognition and elimination of local tumor cells
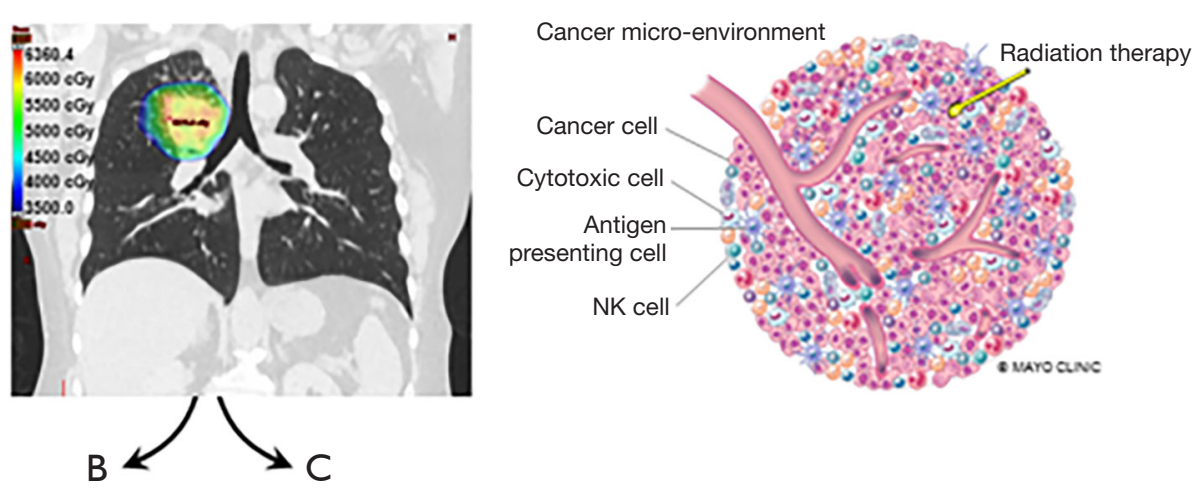

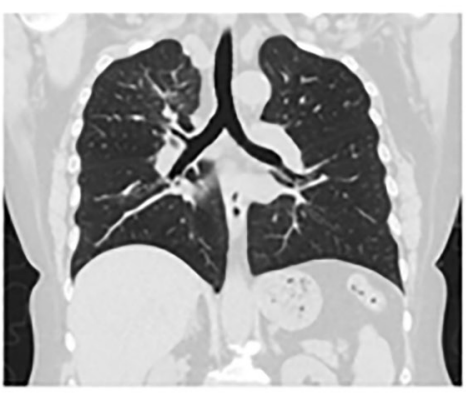

Local tumor effect

- Apoptosis

- Necrosis

- Autophagy

- Mitotic catastrophe

- Proliferative senescence

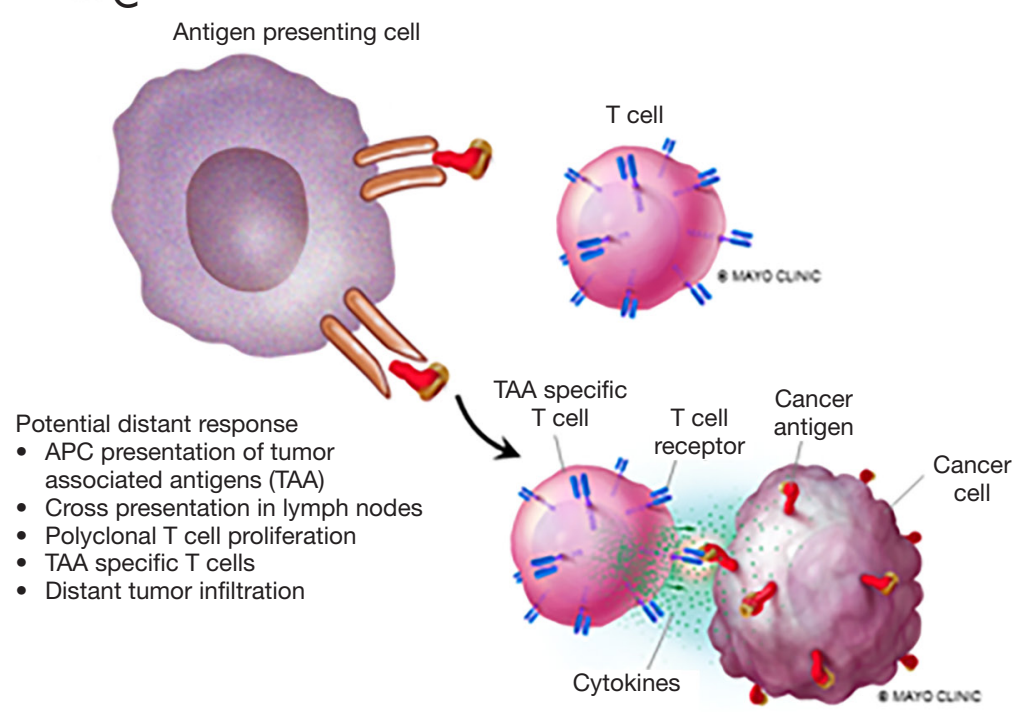

Figure 2 The immunogenic impact of radiation therapy. Local immune effects caused by radiation therapy (A) can provide local tumor control (B) and potentially aid in distant tumor control (C).

treatment to only one site of disease, with hopes of eliciting a response at distant sites, Brooks and Chang provide compelling arguments for treating multiple or all sites of disease (45). When treating a single site of disease, distant response at untreated lesions (or abscopal effect) is dependent upon the same tumor-related antigens released from the radiated site being present on distant sites of disease, as well as immune cell access to distant tumors. However, if multiple or all sites of clinically apparent disease are radiated, more tumor related antigens are released, more inflammation and vascular changes are induced, and cancer is more optimally debulked. Tumor debulking is an attractive target in treating metastatic cancer, as increased volume of disease may have an immunosuppressive effect (46).

While the reasons described above provide motivation to investigate multi-site radiation in the context of IO, concerns exist regarding the potential toxicities of this approach, particularly in a metastatic population treated with ostensibly non-curative intent. On three prospective studies of consolidative RT for advanced non-small lung cancer, grade $3+$ toxicities were acceptably low, ranging from $13-34 \%$. In the Gomez trial of local consolidate therapy (LCT) versus maintenance therapy or observation, $20 \%$ of patients who received LCT experienced toxicity compared to $16.6 \%$ of patients who received maintenance systemic therapy (47). On the PEMBRO-RT trial, of 35 patients on the SBRT + IO arm, 12 grade 3+ toxicities were reported. The most common adverse events were pneumonia $(n=4)$ and dyspnea $(n=4)(48)$. On another phase II trial, 29 patients with advanced lung cancer treated with SBRT followed by maintenance chemotherapy had only 4 grade $3+$ toxicities (49). A review of studies of RT to any extracranial disease with IO showed grade $3+$ toxicity rates of $7-31 \%$ (50). The authors of the review argue that 
combining RT to IO does not increase toxicity over either modality alone, though this has not yet been prospectively validated. Ultimately, this potential for toxicity when combining multisite RT with IO underlies the need for optimal patient selection for aggressive treatment. We await the results of NRG LU002 (NCT03137771) to provide more definitive answers to these questions.

\section{Radiation timing}

In the landmark PACIFIC trial discussed below, Durvalumab delivered after CRT led to unprecedented survival for patients with unresectable stage III lung cancer (28). Durvalumab was administered up to 42 days after completion of CRT, but patients who initiated Durvalumab within 14 days of completing CRT experienced improved survival compared to those who started Durvalumab later. Based on this information, RTOG 3515 requires Durvalumab to be delivered within 7 days of completion of SBRT (NCT03833154). Similarly, on PEMBRO-RT, IO was given within 7 days of completing SBRT. In support of this practice, a mouse study by Dovedi et al. reported a decrease in PD-L1 expression and anergy of tumor-reactive T-cells 7 days after the last dose of fractionated RT (51).

Shaverdian $e t$ al. analyzed patients who received RT at a median of 9.5 months prior to IO on the KEYNOTE-01 clinical trial, and demonstrated that these patients had longer OS and PFS compared to patients who did not receive RT (52). This could indicate that the synergistic benefit of radiation may be long-lasting, though it is not clear if these results would be further improved if the radiation had been delivered closer to the time of IO. Other trials will examine IO delivered before and during RT. Many patients with metastatic disease initiate IO first, and then receive RT at the time of progression. For any of these situations, the optimal time frame remains unclear, but there is likely an ideal sequencing to maximize synergy when delivering IO and RT. For treatment naive patients, initiating IO within one week of completing RT may lead to improved responses, and represents a potential standard practice until more data is available $(28,48,53)$.

\section{Clinical studies evaluating immunotherapy and radiation therapy}

\section{Clinical trials of metastatic disease}

The PD-1/PDL-1 pathway is one of the most studied pathways of immune escape. In some cases, IO alone has become the standard of care over cytotoxic chemotherapy (54). However, durable tumor regression and long-term survival in advanced cancer is only realized in a subset of these patients, with a median progression free survival (PFS) of 10.3 months in patients receiving Pembrolizumab alone for advanced NSCLC (54). In fact, while there is improvement in survival relative to other forms of systemic agents, the vast majority of patients with advanced NSCLC will have short-lived tumor regression with ultimate systemic progression and death. This is likely due to poor recognition of tumor antigens and developed primary resistance to IO.

As RT has the potential to increase tumor antigen release, immune infiltration, and recognition, and is utilized in nearly $50 \%$ of all cancer patients, immune modulation with RT is a heavily researched topic (55). In support of this theory, Shaverdian et al. evaluated patients with NSCLC who received RT prior to receipt of Pembrolizumab on the KEYNOTE 1 clinical trial (52). They found compelling improvements in PFS and OS for patients who received RT (median OS 10.7 months with RT vs. 5.3 months without RT). While this retrospective review of a single arm trial has clear limitations, it raised interest in further investigation of RT and IO.

Bauml et al. performed a single arm clinical trial of local ablative therapy for oligometastatic NSCLC, which included SBRT, CRT, surgery, or percutaneous ablation, followed by Pembrolizumab (56). A total of 51 patients with oligometastatic ( $<4$ metastatic lesions) lung cancer were included. They observed an improvement in PFS from a historical control of 6.6 to 19.1 months $(\mathrm{P}=0.005)$. Median OS was 41.6 months. Due to small numbers and nearly all patients receiving $\mathrm{RT}$, no variables were associated with clinical outcomes. However, given substantial improvements relative to clinical trials with use of IO alone, these findings also led to the addition of RT in future study designs.

PEMBRO-RT is a Dutch Phase II randomized clinical trial of 92 patients with advanced NSCLC treated with Pembrolizumab with or without SBRT (48). Patients who were randomized to SBRT received 24 Gy in 3 fractions to a single site of metastatic NSCLC, followed by standard administration of Pembrolizumab. A separate tumor (not receiving radiation) was measured for objective response rate (ORR), defined as complete or partial response. The primary objective of the trial was to determine whether SBRT improved the ORR at 12 weeks relative to Pembrolizumab alone. They estimated an improvement from $20 \%$ ORR to $50 \%$ ORR with the addition of SBRT. 


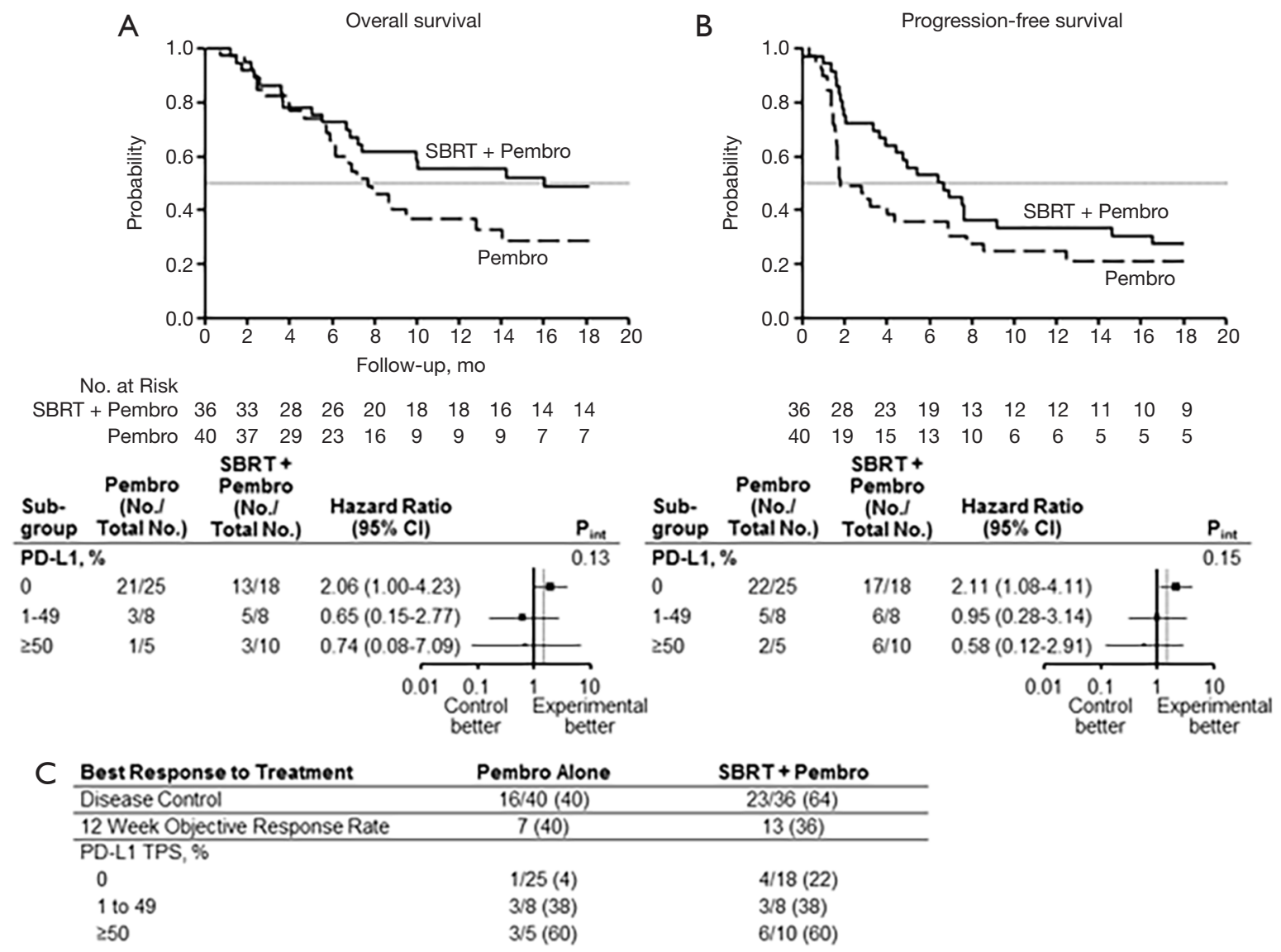

Figure 3 Results from the PEMBRO-RT trial (48). Overall survival (A), Progression-free survival (B), and objective response rate (C) for pembrolizumab (pembro) vs. stereotactic body radiation therapy + pembrolizumab (SBRT + pembro). Mo, months; No, number; TPS, tumor proportion score; SBRT, stereotactic body radiation therapy.

At 12 weeks, the ORR was doubled in patients who received SBRT, at $36 \%$ in the investigational arm vs. $18 \%$ in the control arm $(\mathrm{P}=0.07)$. Patients who received SBRT were more likely to have a complete response $(\mathrm{n}=3$ vs. $\mathrm{n}=1)$, partial response $(\mathrm{n}=14 v s . \mathrm{n}=8)$ and less likely to have progressive disease $(\mathrm{n}=10 v s . \mathrm{n}=21)$. Patients who received SBRT had a non-significant improvement in both median PFS (1.9 vs. 6.6 months, (hazard ratio: 0.71; 95\% CI: $0.42-$ 1.18; $\mathrm{P}=0.19)$ and $\mathrm{OS}(7.6$ vs. 15.9 months, hazard ratio: 0.66; 95\% CI: $0.37-1.18 ; \mathrm{P}=0.16$ ) (Figure $3 A, B$ ).

Interestingly, the numeric improvements in ORR, PFS, and OS in the SBRT group were largely driven by the subgroup of patients with $0 \% \mathrm{PD}-\mathrm{L} 1$ staining. In fact, $22 \%$ of patients with $0 \% \mathrm{PD}-\mathrm{L} 1$ staining had a response in the SBRT group vs. only $4 \%$ in the Pembrolizumab alone group (Figure 3C). When compared to KEYNOTE-010 and -001 , these patients who received SBRT had a numerically greater ORR $(52,57)$. Both PFS and OS were significantly improved with SBRT in the PD-L1 negative group $(57,58)$. As PD-L1 staining is shown to be a predictor of response to IO, this finding may be of substantial importance in selecting appropriate candidates for SBRT with IO: patients with low PD-L1 expression may have improved responses to IO after receiving SBRT. Importantly, no increase in substantial toxic events was observed in the SBRT arm. While there are several limitations to the study, it is the first of its kind showing improvement in distant response rates with localized RT added to IO in a well-designed randomized clinical trial.

\section{Clinical trials of non-metastatic disease}

In 2018, the FDA approved Durvalumab, a PD-L1 inhibitor, after the PACIFIC clinical trial demonstrated its efficacy 
Table 2 Selected current clinical trials of immunotherapy for locally advanced lung cancer

\begin{tabular}{lccccc}
\hline Study & Drug & Trial Phase & Treatment Arm(s) & Enrollment (actual or planned) & Primary Endpoint \\
\hline $\begin{array}{l}\text { NCT02434081 } \\
\text { (NICOLAS) }\end{array}$ & Nivolumab & 2 & CRT +/- concurrent Nivolumab & 94 & Grade 3+ pneumonitis \\
NCT03519971 & Durvalumab & 3 & CRT +/- concurrent Durvalumab & 328 & PFS and ORR \\
\hline
\end{tabular}

CRT, chemoradiation therapy; PFS, progression-free survival; ORR, objective response rate.

following chemoradiation (CRT) compared to placebo in 713 patients with node positive NSCLC $(28,59)$. Patients who received Durvalumab after completion of CRT had significant improvements in PFS and OS. The median PFS for patients who received Durvalumab was 17.2 months, compared to 5.6 months with placebo. The median time to death has not been reached in the Durvalumab group (lower bound $95 \% \mathrm{CI}$ is 36 months), compared to 28.7 months in the placebo group. Patients on the investigational arm were more likely to have further objective response in the thorax, longer duration of response, and a lower likelihood of distant metastasis.

PD-L1 testing was an optional component of PACIFIC. Of 451 patients with evaluable tissue, $67 \%$ had PD-L1 tumor cell staining $\geq 1 \%$. On subset analysis, PFS and OS favored Durvalumab over placebo in patients with PD-L1 staining $\geq 1 \%$ and those with unknown values. Patients with $<1 \%$ staining, which represented $20 \%$ of all enrolled patients, did not have a statistically significant improvement in clinical endpoints with Durvalumab. While this incomplete post-hoc analysis is only hypothesis generating, it is consistent with previous data indicating worse response in tumors with lower PD-L1 expression (57). As discussed above, this obstacle may be overcome in some situations by adding SBRT prior to or during IO, as PEMBRO-RT demonstrated that SBRT followed by IO may be of greatest benefit in inducing response in patients with initially low PD-L1 expression (48).

While adjuvant Durvalumab is standard of care for stage III lung cancer after CRT, other clinical trials are building upon the results of the PACIFIC trial and evaluating alternative timing of IO administration (concurrent $v$ s. sequential), novel IO drugs, varied radiation dose and fractionation, and elimination of chemotherapy (Table 2).

\section{Early stage lung cancer}

SBRT is a standard of care therapy for patients with node negative, early stage lung cancer who are medically inoperable or decline surgery (60). While local control rates exceed $90 \%$, patients are still at risk of nodal and distant relapse, as well as new primary lung cancers (61). Unlike in locally advanced lung cancer, no systemic therapy during or after SBRT for early stage lung cancer is currently standard of care. Given improvements in objective response rates at the primary tumor and reduction in metastatic progression in stage III lung cancer, IO after completion of SBRT for node negative lung cancer is an active area of interest. In particular, given the potential for a more immunogenic response to SBRT compared to standard fractionation CRT, there is hope that adding IO to SBRT will improve outcomes and change patterns of failure for early stage lung cancer. Several clinical trials are ongoing utilizing various IO drugs (Table 3).

\section{Patient selection for future clinical trials}

In stage III lung cancer, Durvalumab is first line therapy for all patients after completion of CRT, and thus all patients receive both RT and IO. As described above, multiple clinical trials are ongoing to evaluate the role of IO with SBRT in early stage lung cancer, though this treatment is confined to clinical trials.

For metastatic lung cancer, in which upfront IO is standard of care, it remains a critical challenge to determine which patients should or should not receive RT in addition to IO. For patients with symptomatic lesions, addition of palliative RT is indicated and often delivered before or concurrently with IO. More challenging is determining which patients with asymptomatic metastatic lesions may benefit systemically from localized RT.

Integration of biomarkers to predict response and appropriately select patients for IO with RT is of the highest importance in the development of clinical trials. PD-L1 is a standard tumor marker predictive of response to IO, as well as a decision point in treatment recommendations for patients with advanced NSCLC (46,57,62-66). Still, PD-L1 assessment is not without limitations, including 
Table 3 Selected Current Clinical Trials of Immunotherapy for Early Stage Lung Cancer

\begin{tabular}{lccccc}
\hline Study & Drug & Trial Phase & Treatment Arm(s) & Enrollment (actual or planned) & Primary Endpoint \\
\hline $\begin{array}{l}\text { NCT03833154 } \\
\text { (PACIFIC-4) }\end{array}$ & Durvalumab & 3 & SBRT +/- adjuvant Durvalumab & 706 & PFS \\
$\begin{array}{l}\text { NCT03110978 (I-SABR) } \\
\text { NCT03924869 }\end{array}$ & Nivolumab & 2 & SBRT +/- adjuvant Nivolumab & 140 & EFS \\
(KEYNOTE-867) & Pembrolizumab & 3 & $\begin{array}{l}\text { SBRT +/- concurrent and } \\
\text { adjuvant Pembrolizumab }\end{array}$ & 530 & OS and EFS \\
\hline
\end{tabular}

SBRT, stereotactic body radiation therapy; PFS, progression-free survival; EFS, event-free survival; OS, overall survival.

heterogeneity within tumors and changing expression over time. Accordingly, other biomarkers, such as TMB, have been explored. Tumors with more mutations are more likely to express novel neo-peptides, leading to subsequent immune activation. TMB has been correlated with patient response to both CTLA-4 and PD-1/PD-L1 blockade. In one study, TMB was a greater predictor of IO response than PD-L1 expression (67-69). Cancers with indications for IO, including NSCLC, SCLC, and melanoma, have high TMB relative to other cancer types. Several other tumor signatures based on gene expression are under current investigation (70).

To date, PEMBRO-RT is the most compelling clinical data demonstrating improvements in objective response rates with the addition of RT to IO. Interestingly, the benefit of RT was primarily in patients with no tumor cell PD-L1 expression. While this does not necessarily mean all patients with negative PD-L1 expression should receive RT, it should be an active area of clinical research to determine when the addition of RT may improve clinical outcomes in this patient population. Indeed, clinical trials should focus on using RT as a stimulator of the immune system, particularly in patients who are unlikely to respond to IO, or have developed resistance to IO.

It is likely that RT will be harnessed more frequently as a means to enhance the efficacy of IO for patients with advanced or metastatic lung cancer, by inducing a systemic response from a localized treatment. For now, and until additional level 1 evidence emerges, patients are best served with treatment on a well-designed clinical trial.

\section{Conclusions}

In the era of IO, lung cancer patients are experiencing unprecedented cancer control and overall survival. The potential for RT to serve as an immunomodulator improving the efficacy of IO could lead to further improvements in outcomes, potentially curing patients with advanced and metastatic lung cancer.

\section{Acknowledgments}

Funding: None.

\section{Footnote}

Provenance and Peer Review: This article was commissioned by the Guest Editor (Chi Wan Koo) for the series "Contemporary Practice in Thoracic Neoplasm Diagnosis, Evaluation and treatment" published in fournal of Thoracic Disease. The article was sent for external peer review organized by the Guest Editor and the editorial office.

Conflicts of Interest: All authors have completed the ICMJE uniform disclosure form, available at: http://dx.doi. org/10.21037/jtd-2019-cptn-07. The series "Contemporary Practice in Thoracic Neoplasm Diagnosis, Evaluation and Treatment" was commissioned by the editorial office without any funding or sponsorship. KL reports honoraria for the institution from OncLive, other from Takeda, outside the submitted work. KWM reports grants from Astra Zeneca, grants from Advanced Accelerator Applications, and grants from Pfizer, outside the submitted work. The other authors have no other conflicts of interest to declare.

Ethical Statement: The authors are accountable for all aspects of the work in ensuring that questions related to the accuracy or integrity of any part of the work are appropriately investigated and resolved.

Open Access Statement: This is an Open Access article distributed in accordance with the Creative Commons Attribution-NonCommercial-NoDerivs 4.0 International 
License (CC BY-NC-ND 4.0), which permits the noncommercial replication and distribution of the article with the strict proviso that no changes or edits are made and the original work is properly cited (including links to both the formal publication through the relevant DOI and the license). See: https://creativecommons.org/licenses/by-nc-nd/4.0/.

\section{References}

1. Male D, Brostoff J, Roth D, et al. Cells, Tissues, and Organs of the Immune System. In: Immunology. 8th Edition. Elsevier, 2013:17-30.

2. Baumeister SH, Freeman GJ, Dranoff G, et al. Coinhibitory Pathways in Immunotherapy for Cancer. Annu Rev Immunol 2016;34:539-73.

3. Walker LS, Sansom DM. The emerging role of CTLA4 as a cell-extrinsic regulator of $\mathrm{T}$ cell responses. Nat Rev Immunol 2011;11:852-63.

4. Leach DR, Krummel MF, Allison JP. Enhancement of antitumor immunity by CTLA-4 blockade. Science 1996;271:1734-6.

5. Klocke K, Sakaguchi S, Holmdahl R, et al. Induction of autoimmune disease by deletion of CTLA-4 in mice in adulthood. Proc Natl Acad Sci U S A 2016;113:E2383-92.

6. Kinter AL, Godbout EJ, McNally JP, et al. The common gamma-chain cytokines IL-2, IL-7, IL-15, and IL-21 induce the expression of programmed death-1 and its ligands. J Immunol 2008;181:6738-46.

7. Francisco LM, Salinas VH, Brown KE, et al. PDL1 regulates the development, maintenance, and function of induced regulatory T cells. J Exp Med 2009;206:3015-29.

8. Luo L, Chapoval AI, Flies DB, et al. B7-H3 enhances tumor immunity in vivo by costimulating rapid clonal expansion of antigen-specific CD8+ cytolytic T cells. J Immunol 2004;173:5445-50.

9. Schreiber RD, Old LJ, Smyth MJ. Cancer immunoediting: integrating immunity's roles in cancer suppression and promotion. Science 2011;331:1565-70.

10. Alexandrov LB, Nik-Zainal S, Wedge DC, et al. Signatures of mutational processes in human cancer. Nature 2013;500:415-21.

11. Lawrence MS, Stojanov P, Polak P, et al. Mutational heterogeneity in cancer and the search for new cancerassociated genes. Nature 2013;499:214-8.

12. Iwai $Y$, Ishida $M$, Tanaka $Y$, et al. Involvement of PD-L1 on tumor cells in the escape from host immune system and tumor immunotherapy by PD-L1 blockade. Proc Natl
Acad Sci U S A 2002;99:12293-7.

13. Mu CY, Huang JA, Chen Y, et al. High expression of PD-L1 in lung cancer may contribute to poor prognosis and tumor cells immune escape through suppressing tumor infiltrating dendritic cells maturation. Med Oncol 2011;28:682-8.

14. Baskar R, Lee KA, Yeo R, et al. Cancer and radiation therapy: current advances and future directions. Int J Med Sci 2012;9:193-9.

15. Chakraborty M, Abrams SI, Camphausen K, et al. Irradiation of tumor cells up-regulates Fas and enhances CTL lytic activity and CTL adoptive immunotherapy. J Immunol 2003;170:6338-47.

16. Demaria S, Golden EB, Formenti SC. Role of Local Radiation Therapy in Cancer Immunotherapy. JAMA Oncol 2015;1:1325-32.

17. Galluzzi L, Kepp O, Kroemer G. Immunogenic cell death in radiation therapy. Oncoimmunology 2013;2:e26536.

18. Golden EB, Pellicciotta I, Demaria S, et al. The convergence of radiation and immunogenic cell death signaling pathways. Front Oncol 2012;2:88.

19. Lugade AA, Sorensen EW, Gerber SA, et al. Radiationinduced IFN-gamma production within the tumor microenvironment influences antitumor immunity. J Immunol 2008;180:3132-9.

20. Matsumura S, Wang B, Kawashima N, et al. Radiationinduced CXCL16 release by breast cancer cells attracts effector T cells. J Immunol 2008;181:3099-107.

21. Reits EA, Hodge JW, Herberts CA, et al. Radiation modulates the peptide repertoire, enhances MHC class I expression, and induces successful antitumor immunotherapy. J Exp Med 2006;203:1259-71.

22. Stone HB, Coleman CN, Anscher MS, et al. Effects of radiation on normal tissue: consequences and mechanisms. Lancet Oncol 2003;4:529-36.

23. Torihata H, Ishikawa F, Okada Y, et al. Irradiation upregulates CD80 expression through two different mechanisms in spleen B cells, B lymphoma cells, and dendritic cells. Immunology 2004;112:219-27.

24. Quarmby S, Hunter RD, Kumar S. Irradiation induced expression of CD31, ICAM-1 and VCAM-1 in human microvascular endothelial cells. Anticancer Res 2000;20:3375-81.

25. Mole RH. Whole body irradiation; radiobiology or medicine? Br J Radiol 1953;26:234-41.

26. Siva S, Callahan J, MacManus MP, et al. Abscopal [corrected] effects after conventional and stereotactic lung irradiation of non-small-cell lung cancer. J Thorac Oncol 
2013;8:e71-2.

27. Golden EB, Chhabra A, Chachoua A, et al. Local radiotherapy and granulocyte-macrophage colonystimulating factor to generate abscopal responses in patients with metastatic solid tumours: a proof-of-principle trial. Lancet Oncol 2015;16:795-803.

28. Antonia SJ, Villegas A, Daniel D, et al. Overall Survival with Durvalumab after Chemoradiotherapy in Stage III NSCLC. N Engl J Med 2018;379:2342-50.

29. Ball D, Mai GT, Vinod S, et al. Stereotactic ablative radiotherapy versus standard radiotherapy in stage 1 nonsmall-cell lung cancer (TROG 09.02 CHISEL): a phase 3 , open-label, randomised controlled trial. Lancet Oncol 2019;20:494-503.

30. Venkatesulu BP, Mallick S, Lin SH, et al. A systematic review of the influence of radiation-induced lymphopenia on survival outcomes in solid tumors. Crit Rev Oncol Hematol 2018;123:42-51.

31. Lee Y, Auh SL, Wang Y, et al. Therapeutic effects of ablative radiation on local tumor require $\mathrm{CD} 8+\mathrm{T}$ cells: changing strategies for cancer treatment. Blood 2009;114:589-95.

32. Mehrazin R, Uzzo RG, Kutikov A, et al. Lymphopenia is an independent predictor of inferior outcome in papillary renal cell carcinoma. Urol Oncol 2015;33:388.e19-25.

33. Wild AT, Ye X, Ellsworth SG, et al. The Association Between Chemoradiation-related Lymphopenia and Clinical Outcomes in Patients With Locally Advanced Pancreatic Adenocarcinoma. Am J Clin Oncol 2015;38:259-65.

34. Crocenzi T, Cottam B, Newell P, et al. A hypofractionated radiation regimen avoids the lymphopenia associated with neoadjuvant chemoradiation therapy of borderline resectable and locally advanced pancreatic adenocarcinoma. J Immunother Cancer 2016;4:45.

35. Pagès F, Galon J, Dieu-Nosjean MC, et al. Immune infiltration in human tumors: a prognostic factor that should not be ignored. Oncogene 2010;29:1093-102.

36. Park HJ, Griffin RJ, Hui S, et al. Radiation-induced vascular damage in tumors: implications of vascular damage in ablative hypofractionated radiotherapy (SBRT and SRS). Radiat Res 2012;177:311-27.

37. Brooks ED, Schoenhals JE, Tang C, et al. Stereotactic Ablative Radiation Therapy Combined With Immunotherapy for Solid Tumors. Cancer J 2016;22:257-66.

38. Ko EC, Formenti SC. Radiotherapy and checkpoint inhibitors: a winning new combination? Ther Adv Med
Oncol 2018;10:1758835918768240.

39. Dewan MZ, Galloway AE, Kawashima N, et al. Fractionated but not single-dose radiotherapy induces an immune-mediated abscopal effect when combined with anti-CTLA-4 antibody. Clin Cancer Res 2009;15:5379-88.

40. Salama JK, Chmura SJ, Mehta N, et al. An initial report of a radiation dose-escalation trial in patients with one to five sites of metastatic disease. Clin Cancer Res 2008;14:5255-9.

41. Twyman-Saint Victor C, Rech AJ, Maity A, et al. Radiation and dual checkpoint blockade activate nonredundant immune mechanisms in cancer. Nature 2015;520:373-7.

42. NCI. Study of Combined Ionizing Radiation and Ipilimumab in Metastatic Non-small Cell Lung Cancer (NSCLC). 2014. Accessed 3/10/2020 2020. Available online: https://clinicaltrials.gov/ct2/show/ NCT02221739

43. Klug F, Prakash H, Huber PE, et al. Low-dose irradiation programs macrophage differentiation to an iNOS(+)/ M1 phenotype that orchestrates effective $T$ cell immunotherapy. Cancer Cell 2013;24:589-602.

44. Gupta S, Koru-Sengul T, Arnold SM, et al. Low-dose fractionated radiation potentiates the effects of cisplatin independent of the hyper-radiation sensitivity in human lung cancer cells. Mol Cancer Ther 2011;10:292-302.

45. Brooks ED, Chang JY. Time to abandon single-site irradiation for inducing abscopal effects. Nature reviews Clinical oncology 2019;16:123-35.

46. Choi SH, Stuckey DW, Pignatta S, et al. Tumor Resection Recruits Effector T Cells and Boosts Therapeutic Efficacy of Encapsulated Stem Cells Expressing IFNbeta in Glioblastomas. Clin Cancer Res 2017;23:7047-58.

47. Gomez DR, Blumenschein GR, Jr., Lee JJ, et al. Local consolidative therapy versus maintenance therapy or observation for patients with oligometastatic non-smallcell lung cancer without progression after first-line systemic therapy: a multicentre, randomised, controlled, phase 2 study. The Lancet Oncology 2016;17:1672-82.

48. Theelen WSME, Peulen HMU, Lalezari F, et al. Effect of Pembrolizumab After Stereotactic Body Radiotherapy vs Pembrolizumab Alone on Tumor Response in Patients With Advanced Non-Small Cell Lung Cancer: Results of the PEMBRO-RT Phase 2 Randomized Clinical Trial. JAMA Oncol 2019;5:1276-82.

49. Iyengar P, Wardak Z, Gerber DE, et al. Consolidative Radiotherapy for Limited Metastatic Non-Small-Cell Lung Cancer: A Phase 2 Randomized Clinical Trial. JAMA 
Oncol 2018;4:e173501.

50. Hwang WL, Pike LRG, Royce TJ, et al. Safety of combining radiotherapy with immune-checkpoint inhibition. Nature reviews Clinical oncology 2018;15:477-94.

51. Dovedi SJ, Melis MH, Wilkinson RW, et al. Systemic delivery of a TLR7 agonist in combination with radiation primes durable antitumor immune responses in mouse models of lymphoma. Blood 2013;121:251-9.

52. Shaverdian N, Lisberg AE, Bornazyan K, et al. Previous radiotherapy and the clinical activity and toxicity of pembrolizumab in the treatment of non-small-cell lung cancer: a secondary analysis of the KEYNOTE-001 phase 1 trial. Lancet Oncol 2017;18:895-903.

53. Young KH, Baird JR, Savage T, et al. Optimizing Timing of Immunotherapy Improves Control of Tumors by Hypofractionated Radiation Therapy. PLoS One 2016;11:e0157164.

54. Reck M, Rodriguez-Abreu D, Robinson AG, et al. Pembrolizumab versus Chemotherapy for PD-L1Positive Non-Small-Cell Lung Cancer. N Engl J Med 2016;375:1823-33.

55. Atun R, Jaffray DA, Barton MB, et al. Expanding global access to radiotherapy. Lancet Oncol 2015;16:1153-86.

56. Bauml JM, Mick R, Ciunci C, et al. Pembrolizumab After Completion of Locally Ablative Therapy for Oligometastatic Non-Small Cell Lung Cancer: A Phase 2 Trial. JAMA Oncol 2019;5:1283-90.

57. Herbst RS, Baas P, Kim DW, et al. Pembrolizumab versus docetaxel for previously treated, PD-L1-positive, advanced non-small-cell lung cancer (KEYNOTE-010): a randomised controlled trial. Lancet 2016;387:1540-50.

58. Garon EB, Rizvi NA, Hui R, et al. Pembrolizumab for the treatment of non-small-cell lung cancer. N Engl J Med 2015;372:2018-28.

59. Antonia SJ, Villegas A, Daniel D, et al. Durvalumab after Chemoradiotherapy in Stage III Non-Small-Cell Lung Cancer. N Engl J Med 2017;377:1919-29.

60. Non-Small Cell Lung Cancer. In: NCCN Clinic Practice Guidelines in Oncology. Accessed March 14, 2020. Available online: https://www.nccn.org/professionals/

Cite this article as: Breen WG, Leventakos K, Dong H, Merrell KW. Radiation and immunotherapy: emerging mechanisms of synergy. J Thorac Dis 2020;12(11):7011-7023. doi: 10.21037/jtd-2019-cptn-07 physician_gls/pdf/nscl.pdf

61. Timmerman RD, Hu C, Michalski JM, et al. Longterm Results of Stereotactic Body Radiation Therapy in Medically Inoperable Stage I Non-Small Cell Lung Cancer. JAMA Oncol 2018;4:1287-8.

62. Leighl NB, Hellmann MD, Hui R, et al. Pembrolizumab in patients with advanced non-small-cell lung cancer (KEYNOTE-001): 3-year results from an open-label, phase 1 study. Lancet Respir Med 2019;7:347-57.

63. Paz-Ares L, Luft A, Vicente D, et al. Pembrolizumab plus Chemotherapy for Squamous Non-Small-Cell Lung Cancer. N Engl J Med 2018;379:2040-51.

64. Gandhi L, Rodriguez-Abreu D, Gadgeel S, et al. Pembrolizumab plus Chemotherapy in Metastatic Non-Small-Cell Lung Cancer. N Engl J Med 2018;378:2078-92.

65. Langer CJ, Gadgeel SM, Borghaei H, et al. Carboplatin and pemetrexed with or without pembrolizumab for advanced, non-squamous non-small-cell lung cancer: a randomised, phase 2 cohort of the open-label KEYNOTE-021 study. Lancet Oncol 2016;17:1497-508.

66. Socinski MA, Jotte RM, Cappuzzo F, et al. Atezolizumab for First-Line Treatment of Metastatic Nonsquamous NSCLC. N Engl J Med 2018;378:2288-301.

67. Rizvi NA, Hellmann MD, Snyder A, et al. Cancer immunology. Mutational landscape determines sensitivity to PD-1 blockade in non-small cell lung cancer. Science 2015;348:124-8.

68. Rosenberg JE, Hoffman-Censits J, Powles T, et al. Atezolizumab in patients with locally advanced and metastatic urothelial carcinoma who have progressed following treatment with platinum-based chemotherapy: a single-arm, multicentre, phase 2 trial. Lancet 2016;387:1909-20.

69. Snyder A, Makarov V, Merghoub T, et al. Genetic basis for clinical response to CTLA-4 blockade in melanoma. $\mathrm{N}$ Engl J Med 2014;371:2189-99.

70. Lu S, Stein JE, Rimm DL, et al. Comparison of Biomarker Modalities for Predicting Response to PD-1/PD-L1 Checkpoint Blockade: A Systematic Review and Metaanalysis. JAMA Oncol 2019;5:1195-204. 